Nambu, T. and Sakawa, Y.

Osaka J. Math.

14 (1977), 333-354

\title{
ASYMPTOTIC BEHAVIOR OF SOLUTIONS OF A CLASS OF NONLINEAR DIFFERENTIAL EQUATIONS IN BANACH SPACE
}

\author{
TAKao NAMBU and Yoshiyuki SAKAWA
}

(Received March 25, 1976)

\section{Introduction}

We consider in this paper the problems of existence, uniqueness, and asymptotic behavior of solutions of the following semi-linear evolution equation in a Banach space $E$ :

$$
\begin{gathered}
\frac{d}{d t} x(t)=A x(t)+f(t, x(t)), \quad 0<t<+\infty, \\
x(0)=x_{0} .
\end{gathered}
$$

$x(t)$ is said to be a solution of (1.1), if

$$
x(\cdot) \in C([0, \infty) ; E) \cap C^{1}((0, \infty) ; E)
$$

and (1.1) is satisfied.

In (1.1), $A$ is a closed linear operator in $E$ with a domain $D(A)$ which is not necessarily dense in $E$. Throughout this paper, it is assumed that the resolvent $R(\mu)=(A-\mu I)^{-1}$ exists and satisfies the estimate

$$
\|R(\mu)\| \leqq \frac{M_{0}}{(1+|\operatorname{Im} \mu|)^{\beta}}
$$

for $\operatorname{Re} \mu \geqq-\lambda$, where $\lambda$ is a constant, and $\beta=(1+\alpha)^{-1}, 0<\alpha<1$.

W. von Wah] [9] and H. Kielhöfer [3] studied the parabolic initial boundary value problems, and obtained the similar estimate to (1.2) (see Section 6).

For fixed $q, 0<q<1$, let $\Gamma_{q}$ be the curve

$$
\Gamma_{q}=\left\{\mu=\sigma+i \tau ; \sigma=-\lambda-\frac{q}{M_{0}}(1+|\tau|)^{\beta},-\infty<\tau<+\infty\right\} .
$$

It is easy to see that the resolvent exists in a region situated to the right of the curve $\Gamma_{q}$ and satisfies (1.2) with $M_{0}(1-q)^{-1}$ instead of $M_{0}$ [4].

Under the assumption (1.2), the weakened Cauchy problem of the following unperturbed linear equation 


$$
\frac{d}{d t} x(t)=A x(t), \quad t>0, \quad x(0)=x_{0}
$$

is well-posed on the set $D(A)$ [4], and the solution $x(t)$ of $(1.3)$ is represented as $U(t) x_{0}$ for $t>0$, where $U(t), t>0$, is the semigroup of bounded linear operators given by

$$
U(t)=-\frac{1}{2 \pi i} \int_{\Gamma_{q}} e^{\mu t} R(\mu) d \mu, \quad t>0 .
$$

It follows from (1.4) that $U(t)$ satisfies the estimate [4]

$$
\|U(t)\| \leqq M e^{-\lambda t} t^{-\infty}, \quad t>0
$$

where $M>0$ is some constant.

For the nonlinear term $f(t, x)$, it is assumed that the following condition (i) or (ii) is satisfied:

(i) $f(t, x)$ is continuous on $[0, \infty) \times E$, and the estimate

$$
\|f(t, x)-f(t, y)\| \leqq K\|x-y\|
$$

holds, where $K>0$ is a constant independent of $t, x$, and $y$.

(ii) $f(t, x)$ is continuous on $[0, \infty) \times E$. For each $c>0$ there exists a constant $k(c)>0$ such that the estimate

$$
\|f(t, x)-f(t, y)\| \leqq k(c)\|x-y\|
$$

holds for $t, x$, and $y$ satisfying $t \geqq 0,\|x\| \leqq c,\|y\| \leqq c$.

In the case where $A$ is the infinitesimal generator of $C_{0}$ semigroup, the problem of existence and uniqueness of solutions of semi-linear equations was treated by several authors, for example, by T. Kato [2], and the problem of asymptotic behavior was treated, for example, by A. Pazy [5]. Recently W. von Wahl [9] and H. Kielhöfer [3] considered the local solvability of (1.1) under the same condition as (1.5) and weaker conditions for $f(t, x)$. They also applied their results to a semi-linear initial boundary value problem within the framework of the $C^{a}$-theory. Our main purpose is to obtain an estimate for the asymptotic behavior of the solution of (1.1) and its derivative under the condition (1.5). Some examples of linear partial differential equations are worked out.

\section{Existence and uniqueness of solutions}

In this section it is assumed that $f(t, x)$ satisfies (1.6). We consider the problem of existence and uniqueness of the solution of (1.1) under the condition (1.6).

Let $x(t)$ be a solution of (1.1). Then we have the integral equation 


$$
x(t)=U(t) x_{0}+\int_{0}^{t} U(t-s) f(s, x(s)) d s .
$$

(2.1) can be uniquely solved by the successive approximations:

$$
\begin{aligned}
& x_{0}(t)=U(t) x_{0}, \\
& x_{n+1}(t)=U(t) x_{0}+\int_{0}^{t} U(t-s) f\left(s, x_{n}(s)\right) d s, \quad n=0,1, \cdots, \\
& x(t)=\lim _{n \rightarrow \infty} x_{n}(t) .
\end{aligned}
$$

If $x_{0} \in D(A)$, the solution $x(t)$ of $(2.1)$ is in $C([0, \infty) ; E)$. Otherwise $x(t)$ is in $C((0, \infty) ; E)$ and satisfies the following estimate in the neighbourhood of $t=0$ :

$$
\|x(t)\| \leqq c_{1} t^{-\infty}
$$

where $c_{1}>0$ is some constant.

Next we shall show that the solution of (2.1) is continuously differentiable in $t>0$ by posing some additional assumptions on $f(t, x)$. Suppose that $f(t, x)$ satisfies the following conditions:

(i) $\frac{\partial}{\partial t} f(t, x)=f_{t}(t, x)$ is continuous for $(t, x) \in[0, \infty) \times E$.

(ii) For each $t \geqq 0, f(t, x)$ is Fréchet differentiable in $x$ (Tanabe [8]), i.e.,

$$
f(t, x+z)=f(t, x)+(D f(t, x)+\bar{D} f(t, x)) \cdot z+o(\|z\| ; x)
$$

holds when $\|z\| \rightarrow 0$, where $D f(t, x)$ (resp. $\bar{D} f(t, x)$ ) is a linear (resp. anti-linear) bounded operator in $E$.

(iii) $D f(t, x)$ and $\bar{D} f(t, x)$ are strongly continuous for $(t, x) \in[0, \infty) \times E$.

(iv) For $t, x$ satisfying $0 \leqq t \leqq c,\|x\| \leqq c,\left\|f_{t}(t, x)\right\|,\|D f(t, x)\|_{L(E, E)}$, and $\|\bar{D} f(t, x)\|_{L(E, E)}$ are uniformly bounded.

The following lemma is well-known, if $f(t) \in C^{1}([0, \infty) ; E)$ and $U(t)$ is of $C_{0}$ class.

Lemma 2.1. Suppose that $f(t)$ belongs to $C([0, \infty) ; E) \cap C^{1}((0, \infty) ; E)$. Furthermore, suppose that $\left\|\frac{d}{d t} f(t)\right\|$ is integrable on $(0, T)$, where $T>0$. Then we have for $t>0$

$$
\begin{aligned}
\frac{d}{d t} \int_{0}^{t} U(t-s) f(s) d s & =U(t) f(0)+\int_{0}^{t} U(t-s) \frac{d}{d s} f(s) d s \\
& =A \int_{0}^{t} U(t-s) f(s) d s+f(t) .
\end{aligned}
$$


Proof. If $0 \in \rho(A)$, the resolvent set of $A$, then it is easily seen that

$$
y(t)=\int_{0}^{t} U(t-s) A^{-1} \frac{d}{d s} f(s) d s-A^{-1} f(t)+U(t) A^{-1} f(0)
$$

is in $C([0, \infty) ; E) \cap C^{1}((0, \infty) ; E)$ and satisfies for $t>0$

$$
\begin{aligned}
\frac{d}{d t} y(t) & =A y(t)+f(t) \\
& =U(t) f(0)+\int_{0}^{t} U(t-s) \frac{d}{d s} f(s) d s
\end{aligned}
$$

and $y(0)=0$. Therefore

$$
y(t)=\int_{0}^{t} U(t-s) f(s) d s
$$

holds for $t \geqq 0$.

If $0 \notin \rho(A)$, the relation (2.8) holds for

$$
A_{\sigma}=A-\sigma I, \quad U_{\sigma}(t)=e^{-\sigma t} U(t), \quad f_{\sigma}(t)=e^{-\sigma t} f(t),
$$

where $\sigma \in \rho(A)$. Then we can derive (2.8) by differentiating the following equation in $t$ :

$$
\int_{0}^{t} U(t-s) f(s) d s=e^{\sigma t} \int_{0}^{t} U_{\sigma}(t-s) f_{\sigma}(s) d s .
$$

The following theorem can be proved in the similar way to SobolevskiiPogorelenko [7]:

Theorem 2.2. If $f(t, x)$ satisfies (1.6) and (2.4) to (2.7), then for each $x_{0} \in D(A)$ the solution of $(2.1)$ is continuously differentiable in $t>0$ and satisfies (1.1). Furthermore, the estimate

$$
\left\|\frac{d}{d t} x(t)\right\| \leqq c_{2} t^{-\infty}
$$

holds as $t$ tends to 0 , where $c_{2}$ is a constant.

Proof. By the Fréchet differentiability of $f(t, x)$ and by the fact that $x_{0} \in D(A)$, we obtain for $0<t \leqq T$

$$
\begin{aligned}
\frac{d}{d t} f\left(t, x_{0}(t)\right)= & f_{t}\left(t, x_{0}(t)\right)+D f\left(t, x_{0}(t)\right) \cdot \frac{d}{d t} x_{0}(t) \\
& +\bar{D} f\left(t, x_{0}(t)\right) \cdot \frac{d}{d t} x_{0}(t), \\
& \left\|\frac{d}{d t} f\left(t, x_{0}(t)\right)\right\| \leqq c_{3} t^{-\infty},
\end{aligned}
$$


where $c_{3}$ is a constant. Therefore it follows inductively from Lemma 2.1 that the successive approximations (2.2) satisfy

$$
\begin{aligned}
\frac{d}{d t} x_{n+1}(t)= & U(t) A x_{0}+U(t) f\left(0, x_{0}\right)+\int_{0}^{t} U(t-s)\left[f_{s}\left(s, x_{n}(s)\right)\right. \\
& \left.+D f\left(s, x_{n}(s)\right) \cdot \frac{d}{d s} x_{n}(s)+\bar{D} f\left(s, x_{n}(s)\right) \cdot \frac{d}{d s} x_{n}(s)\right] d s .
\end{aligned}
$$

Consider the integral equation

$$
\begin{aligned}
v(t)= & U(t) A x_{0}+U(t) f\left(0, x_{0}\right)+\int_{0}^{t} U(t-s)\left[f_{s}(s, x(s))\right. \\
& +D f(s, x(s)) \cdot v(s)+\bar{D} f(s, x(s)) \cdot v(s)] d s .
\end{aligned}
$$

The existence and uniqueness of the solution $v(t)$ of $(2.10)$ and the estimate

$$
\|v(t)\| \leqq c_{4} t^{-\infty}, \quad 0<t \leqq T
$$

are ensured by the similar arguments to (2.2). From (2.9) and (2.10) we have

$$
\begin{aligned}
\frac{d}{d t} x_{n+1}(t)-v(t)= & \int_{0}^{t} U(t-s)\left[f_{s}\left(s, x_{n}(s)\right)-f_{s}(s, x(s))\right] d s \\
& +\int_{0}^{t} U(t-s) D f\left(s, x_{n}(s)\right) \cdot\left[\frac{d}{d s} x_{n}(s)-v(s)\right] d s \\
& +\int_{0}^{t} U(t-s)\left[D f\left(s, x_{n}(s)\right)-D f(s, x(s))\right] \cdot v(s) d s \\
& +\int_{0}^{t} U(t-s) \bar{D} f\left(s, x_{n}(s)\right) \cdot\left[\frac{d}{d s} x_{n}(s)-v(s)\right] d s \\
& +\int_{0}^{t} U(t-s)\left[\bar{D} f\left(s, x_{n}(s)\right)-\bar{D} f(s, x(s))\right] \cdot v(s) d s \\
= & \mathrm{I}+\mathrm{II}+\mathrm{III}+\mathrm{IV}+\mathrm{V} .
\end{aligned}
$$

By Hölder's inequality we obtain

$$
\|\mathrm{I}\| \leqq\left\{\int_{0}^{t}\|U(t-s)\|^{p} d s\right\}^{1 / p}\left\{\int_{0}^{t}\left\|f_{s}\left(s, x_{n}(s)\right)-f_{s}(s, x(s))\right\|^{q} d s\right\}^{1 / q},
$$

where $1 / p+1 / q=1$ and $\alpha p<1$. By Lebesgue's dominated convergence theorem $\|\mathrm{I}\|$ converges to 0 uniformly in $t \in(0, T]$. For the third term of $(2.12)$ the estimate

$$
\begin{array}{r}
t^{s}\|\mathrm{III}\| \leqq \int_{0}^{t}(t-s)^{\infty}\|U(t-s)\|\left\|\left[D f\left(s, x_{n}(s)\right)-D f(s, x(s))\right] \cdot v(s)\right\| d s \\
+\left\{\int_{0}^{t}\|U(t-s)\|^{p} d s\right\}^{1 / p}\left\{\int_{0}^{t} \|\left[D f\left(s, x_{n}(s)\right)-D f(s, x(s))\right]\right. \\
\left.\times s^{\infty} v(s) \|^{q} d s\right\}^{1 / q}
\end{array}
$$


holds, where $1 / p+1 / q=1$ and $\alpha p<1$. Similarly $t^{\alpha}|| \mathrm{III} \|$ converges to 0 uniformly in $t \in(0, T]$, and so does $t^{\alpha}\|\mathrm{V}\|$. Consequently there exist $c_{5}>0$ and a sequence of positive numbers $\left\{\varepsilon_{n}\right\}_{n=0}^{\infty}$, such that $\varepsilon_{n} \rightarrow 0$ as $n \rightarrow \infty$, and the estimates

$$
\begin{gathered}
w_{n+1}(t) \leqq \varepsilon_{n} t^{-\infty}+\int_{0}^{t} c_{5}(t-s)^{-\infty} w_{n}(s) d s, \\
t \in(0, T], \quad n=0,1, \cdots
\end{gathered}
$$

hold, where $w_{n}(t)$ are given by

$$
w_{n}(t)=\left\|\frac{d}{d t} x_{n}(t)-v(t)\right\|, \quad t>0 .
$$

From (2.13) it follows inductively that

$$
\begin{gathered}
w_{n+1}(t) \leqq \int_{0}^{t} \frac{\left[c_{5} \Gamma(1-\alpha)\right]^{j+1}}{\Gamma((j+1)(1-\alpha))}(t-s)^{j(1-\infty)-\infty} w_{n-j}(s) d s \\
+\Gamma(1-\alpha) t^{-\infty} \sum_{k=0}^{j} \frac{\left[c_{5} \Gamma(1-\alpha) t^{1-\alpha}\right]^{k}}{\Gamma((k+1)(1-\alpha))} \varepsilon_{n-k} \\
0 \leqq j \leqq n, \quad 0<t \leqq T .
\end{gathered}
$$

Since $w_{0}(t) \leqq c_{6} t^{-\infty}, 0<t \leqq T$, by letting $j=n$ in (2.14), it follows that $t^{\infty} w_{n}(t)$ are uniformly bounded in $t \in(0, T]$ and in $n$, i.e.,

$$
w_{n}(t) \leqq c_{7} t^{-\infty}, \quad t \in(0, T], \quad n=0,1, \cdots .
$$

It follows from (2.14) and (2.15) that

$$
\begin{gathered}
t^{\alpha} w_{n+1}(t) \leqq \\
\Gamma\left(c_{7} \Gamma(1-\alpha)\left[c_{5} \Gamma(1-\alpha) t^{1-\alpha}\right]^{j+1}\right. \\
+\Gamma(1-\alpha) \sum_{k=0}^{j} \frac{\left[c_{5} \Gamma(1-\alpha) t^{1-\alpha}\right]^{k}}{\Gamma((k+1)(1-\alpha))} \varepsilon_{n-k}, \\
0 \leqq j \leqq n, \quad 0<t \leqq T .
\end{gathered}
$$

For any $\varepsilon>0$, choose $j$ sufficiently large so that

$$
\text { [the first term of }(2.16)]<\frac{\varepsilon}{2}, \quad 0 \leqq t \leqq T
$$

holds. Next take a large number $N(\varepsilon)$ so that

$$
\text { [the second term of }(2.16)]<\frac{\varepsilon}{2}, \quad 0 \leqq t \leqq T
$$

holds for any $n>N(\varepsilon)$. Consequently, for any $\delta>0, w_{n}(t)$ converge to 0 uniformly in $t \in[\delta, T]$. Therefore $x(t)$ is continuously differentiable in $t \in(0, T]$ and the following equation holds: 


$$
\begin{aligned}
\frac{d}{d t} x(t) & =U(t) A x_{0}+U(t) f\left(0, x_{0}\right)+\int_{0}^{t} U(t-s) \frac{d}{d s} f(s, x(s)) d s \\
& =A\left[U(t) x_{0}+\int_{0}^{t} U(t-s) f(s, x(s)) d s\right]+f(t, x(t)),
\end{aligned}
$$

where the second equation follows from Lemma 2.1.

Since $\frac{d}{d t} x(t)=v(t)$, the estimate in the Theorem follows from (2.11).

Q.E.D.

\section{Asymptotic behavior I}

Now let us consider the asymptotic behavior of the solution of (1.1) and (2.1). In this section we assume that $f(t, x)$ satisfies (1.6).

It follows from the successive approximations (2.2) that for $0<t \leqq T$

$$
\begin{aligned}
\|x(t)\| \leqq & \sum_{n=1}^{\infty}\left\|x_{n}(t)-x_{n-1}(t)\right\|+\left\|x_{0}(t)\right\| \\
\leqq & \sum_{n=0}^{\infty} \frac{\left[K M \Gamma(1-\alpha) t^{1-\alpha}\right]^{n}}{\Gamma((n+1)(1-\alpha))} M \Gamma(1-\alpha)\left\|x_{0}\right\| e^{-\lambda t} t^{-\infty} \\
+\sup _{0 \leqq s \leqq T}\|f(s, 0)\| M \Gamma(1-\alpha) \int_{0}^{t} \sum_{n=1}^{\infty} \frac{\left[K M \Gamma(1-\alpha) s^{1-\alpha}\right]^{n-1}}{\Gamma(n(1-\alpha))} & \times s^{-\infty} e^{-\lambda s} d s .
\end{aligned}
$$

In the case where $\alpha=0$ and the Cauchy problem of (1.3) is uniformly wellposed, (3.1) is reduced to the inequality

$$
\begin{gathered}
\|x(t)\| \leqq M e^{(K M-\lambda) t}\left\|x_{0}\right\|+\sup _{0 \leqq s \leqq T}\|f(s, 0)\| M \frac{e^{(K M-\lambda) t}-1}{K M-\lambda}, \\
0 \leqq t \leqq T .
\end{gathered}
$$

In particular, if $f(t, x)=B x$, where $B$ is a linear bounded operator in $E$, we have the well-known result [1], [4] from (3.2):

$$
\left\|U_{A+B}(t)\right\| \leqq M \exp \{M\|B\|-\lambda\} t, \quad t \geqq 0,
$$

where $U_{A+B}(t)$ is the semigroup generated by the Cauchy problem

$$
\frac{d}{d t} x(t)=(A+B) x(t), \quad t \geqq 0, \quad x(0)=x_{0} .
$$

Now let us return to (3.2). Suppose that

$$
K M<\lambda, \sup _{0 \leqq s<+\infty}\|f(s, 0)\|<+\infty .
$$

By considering $x\left(t+t_{0}\right)$ instead of $x(t)$ in (3.2), where $t_{0}>0$, we have for $t \geqq 0$ 
Therefore it follows that

$$
\left\|x\left(t+t_{0}\right)\right\| \leqq M e^{-(\lambda-K M) t}\left\|x\left(t_{0}\right)\right\|+\sup _{t_{0} \leqq s}\|f(s, 0)\| M \frac{1-e^{-(\lambda-K M) t}}{\lambda-K M} .
$$

$$
\varlimsup_{t \rightarrow \infty}\|x(t)\| \leqq \frac{M}{\lambda-K M} \sup _{t_{0} \leqq s}\|f(s, 0)\| .
$$

Since $t_{0}$ is arbitrary, from the above inequality we obtain

$$
\varlimsup_{t \rightarrow \infty}\|x(t)\| \leqq \frac{M}{\lambda-K M} \varlimsup_{t \rightarrow \infty}\|f(t, 0)\| .
$$

If $\alpha>0$, it seems difficult to obtain the asymptotic behavior of $x(t)$ from (3.1). In what follows, we derive the similar estimate to (3.3).

The following lemma is easily proved:

Lemma 3.1. Suppose that $f(t)$ is a real valued continuous function of $t \geqq 0$, and that $\varlimsup_{t \rightarrow \infty} f(t)=+\infty$. Then we can choose a sequence $\left\{t_{n}\right\}_{n=1}^{\infty}$ such that

$$
\begin{aligned}
& f(t) \leqq f\left(t_{n}\right)=n, \quad \text { for } \quad 0 \leqq t \leqq t_{n}, \\
& t_{1} \leqq t_{2} \leqq \cdots \leqq t_{n} \leqq \cdots, \quad \lim _{n \rightarrow \infty} t_{n}=+\infty .
\end{aligned}
$$

Proof. Let us define

$$
S_{n}=\{t: f(t) \geqq n, \quad t \geqq 0\} .
$$

By the assumptions the sets $S_{n}$ are closed and nonvoid. Further let

$$
t_{n}=\inf \left\{t: t \in S_{n}\right\} \text {. }
$$

Then $\left\{t_{n}\right\}_{n=1}^{\infty}$ is the sequence stated in the lemma.

Q.E.D.

Now let us prove one of our main results.

Theorem 3.2. Suppose that (1.6) is satisfied and that

$$
\int_{0}^{\infty} K M e^{-\lambda t} t^{-\infty} d t=K M \lambda^{\infty-1} \Gamma(1-\alpha)<1 .
$$

Then we have the following estimate for the solution $x(t)$ of (2.1):

$$
\varlimsup_{t \rightarrow \infty}\|x(t)\| \leqq \frac{M \lambda^{\alpha-1} \Gamma(1-\alpha)}{1-K M \lambda^{\infty-1} \Gamma(1-\alpha)} \varlimsup_{t \rightarrow \infty}\|f(t, 0)\| .
$$

Proof. If $\varlimsup \lim \|f(t, 0)\|=+\infty$, then (3.5) is clear. Therefore we assume henceforth that $\overline{\lim }\|f(t, 0)\|<+\infty$. We set $y(t)=x(t+1)$ and $y_{0}=x(1)$. Then $y(t)$ satisfies (2.1) and continuous in $t \geqq 0$.

First we prove that $\|y(t)\|$ is bounded. If not so, by Lemma 3.1 we can choose a sequence $\left\{t_{n}\right\}_{n=1}^{\infty}$ such that 


$$
\begin{aligned}
n=\left\|y\left(t_{n}\right)\right\| & \leqq\left\|U\left(t_{n}\right) y_{0}\right\|+\int_{0}^{t_{n}}\left\|U\left(t_{n}-s\right)\right\|[K\|y(s)\|+\|f(s+1,0)\|] d s \\
& \leqq\left\|U\left(t_{n}\right) y_{0}\right\|+\sup _{0 \leqq s}\|f(s, 0)\| \int_{0}^{\infty}\|U(s)\| d s+n K \int_{0}^{\infty}\|U(s)\| d s .
\end{aligned}
$$

This is a contradiction by (1.5) and (3.4). Therefore there exists some constant $c_{8}>0$ such that

$$
\|y(t)\| \leqq c_{8}, \quad t \geqq 0 .
$$

Let

$$
a=K M \lambda^{a-1} \Gamma(1-\alpha), \quad b=\varlimsup_{t \rightarrow \infty}\|f(t, 0)\| M \lambda^{a-1} \Gamma(1-\alpha) .
$$

For any $\varepsilon>0$, there exists $T(\varepsilon)>0$ such that the inequality

$$
\int_{0}^{t} M e^{-\lambda(t-s)}(t-s)^{-\infty}\left\|f\left(s+t_{0}, 0\right)\right\| d s \leqq \varepsilon(1-a)+b
$$

holds for any $t_{0} \geqq T(\varepsilon)$, and $t>0$.

Let $x^{0}(t)=x\left(t+t_{0}\right)$, where $t \geqq 0$ and $t_{0} \geqq \max \{1, T(\varepsilon)\}$. Then by (3.6) and (3.7) we have the estimate

$$
\left\|x^{0}(t)\right\| \leqq\left\|U(t) x\left(t_{0}\right)\right\|+c_{8} a+\varepsilon(1-a)+b, \quad t \geqq 0 .
$$

Take $\gamma>0$ such that $a+\gamma<1$. Then, from (1.5) and (3.8), there exists $t_{1}>0$ such that the estimate

$$
\left\|x\left(t+t_{0}\right)\right\|=\left\|x^{0}(t)\right\| \leqq c_{8}(a+\gamma)+\varepsilon(1-a)+b
$$

holds for any $t>t_{1}$. Consequently for any integer $m>0$, we can find inductively $t_{0}, t_{1}, \cdots, t_{m+1}$, such that the estimate

$$
\left\|x\left(t+\sum_{j=0}^{m} t_{j}\right)\right\| \leqq c_{8}(a+\gamma)^{m+1}+[\varepsilon(1-a)+b] \sum_{j=0}^{m} a^{j}
$$

holds for any $t \geqq t_{m+1}$. From (3.10) it can be concluded that there exists $t(\varepsilon)>0$ such that the estimate

$$
\|x(t)\| \leqq 2 \varepsilon+b(1-a)^{-1}
$$

holds for any $t \geqq t(\varepsilon)$. Clearly (3.11) implies that the estimate (3.5) holds.

Q.E.D.

If $f(t, 0)$ tends to 0 when $t \rightarrow \infty$, the following theorem holds:

Theorem 3.3. Suppose that the assumptions of Theorem 3.2 are satisfied, and let $x(t)$ be the solution of (2.1).

(i) If there exist $c>0$ and $\delta>0$ such that the estimate 


$$
\|f(t, 0)\| \leqq c e^{-\delta t}, \quad t \geqq 0
$$

holds, then $x(t)$ satisfies the estimate

$$
\|x(t)\| \leqq \tilde{c} e^{-\tilde{\delta} t} t^{-\infty}, \quad t>0
$$

where $\tilde{c}>0$ and $\tilde{\delta}>0$ are some constants.

(ii) If there exist $c>0$, an integer $n \geqq 0$, and $\beta, 0 \leqq \beta<1$, such that the estimate

$$
\|f(t, 0)\| \leqq c t^{-(n+\beta)}
$$

holds when $t \rightarrow \infty$, then $x(t)$ satisfies the estimate

$$
\|x(t)\| \leqq \widetilde{c} t^{-(n+\beta)}
$$

when $t \rightarrow \infty$, where $\tilde{c}>0$ is some constant.

(iii) If there exists $c>0$ such that the estimate

$$
\|f(t, 0)\| \leqq c\{\ln t\}^{-1}
$$

holds when $t \rightarrow \infty$, then $x(t)$ satisfies the estimate

$$
\|x(t)\| \leqq \tilde{c}\{\ln t\}^{-1}
$$

when $t \rightarrow \infty$, where $\tilde{c}>0$ is some constant.

Proof. (i) Let us take $\nu>0$ so that the inequalities

$$
\nu<\min (\delta, \lambda), \quad K M(\lambda-\nu)^{\alpha-1} \Gamma(1-\alpha)<1
$$

hold. It follows from (2.1) that the estimate

$$
\begin{aligned}
e^{\nu t}\|x(t+1)\| \leqq & e^{\nu t}\|U(t) x(1)\|+\int_{0}^{t} \frac{M e^{-(\lambda-\nu)(t-s)}}{(t-s)^{\alpha}} e^{\nu_{s}}\|f(s+1,0)\| d s \\
& +\int_{0}^{t} \frac{K M e^{-(\lambda-\nu)(t-s)}}{(t-s)^{\alpha}} e^{\nu_{s}}\|x(s+1)\| d s
\end{aligned}
$$

holds for $t>0$. From (1.5), (3.12), and (3.18), the first and the second terms of (3.19) are bounded for $t>0$ and tend to 0 exponentially as $t \rightarrow \infty$. In the same way as Theorem 3.2, (3.18) and (3.19) imply that

$$
e^{\nu t}|| x(t+1) \| \leqq c_{9}, \quad t \geqq 0,
$$

where $c_{9}>0$ is some constant. Clearly the above inequality implies that the estimate (3.13) holds.

(ii) Let $n \geqq 1$. Using the relation $t^{\beta} \leqq(t-s)^{\beta}+s^{\beta}, 0 \leqq s \leqq t, 0 \leqq \beta<1$, we obtain 


$$
\begin{aligned}
& t^{n+\beta} \int_{0}^{t}\|U(t-s)\|\|f(s+1,0)\| d s \\
& \leqq \int_{0}^{t}\|U(t-s)\| \sum_{j=0}^{n}{ }_{n} C_{j}(t-s)^{n-j-\beta} s^{j}\|f(s+1,0)\| d s \\
& \quad+\int_{0}^{t}\|U(t-s)\| \sum_{j=0}^{n}{ }_{n} C_{j}(t-s)^{n-j} s^{j+\beta}\|f(s+1,0)\| d s .
\end{aligned}
$$

It follows from (1.5) and (3.14) that the right-hand side of (3.20) is bounded on $(0, \infty)$.

From (2.1) we obtain the estimate

$$
\begin{aligned}
t\|x(t+1)\| \leqq & t\|U(t) x(1)\|+t \int_{0}^{t}\|U(t-s)\|\|f(s+1,0)\| d s \\
& +\int_{0}^{t} K\|U(t-s)\|(t-s)\|x(s+1)\| d s \\
& +\int_{0}^{t} K\|U(t-s)\| s\|x(s+1)\| d s
\end{aligned}
$$

It follows from (1.5), (3.5), and (3.20) that the first, the second, and the third terms of $(3.21)$ are bounded on $(0, \infty)$. In the same way as Theorem 3.2, (3.21) implies that $t x(t+1)$ is bounded on $(0, \infty)$. It follows inductively that the estimate

$$
t^{n}\|x(t+1)\| \leqq c_{10}, \quad t \geqq 0
$$

holds, where $c_{10}$ is some constant. Consider the inequality

$$
\begin{aligned}
t^{n+\beta}\|x(t+1)\| \leqq & t^{n+\beta}\|U(t) x(1)\|+t^{n+\beta} \int_{0}^{t}\|U(t-s)\|\|f(s+1,0)\| d s \\
& +\int_{0}^{t} K\|U(t-s)\| \sum_{j=0}^{n}{ }_{n} C_{j}(t-s)^{n-j+\beta} s^{j}\|x(s+1)\| d s \\
& +\int_{0}^{t} K\|U(t-s)\| \sum_{j=1}^{n-1}{ }_{n} C_{j}(t-s)^{n-j} s^{j+\beta}\|x(s+1)\| d s \\
& +\int_{0}^{t} K\|U(t-s)\| s^{n+\beta}\|x(s+1)\| d s, \quad t>0 .
\end{aligned}
$$

From (1.5), (3.20), and (3.22), the same argument as Theorem 3.2 implies that $x(t)$ satisfies the estimate (3.15) when $t \rightarrow \infty$.

In the case where $n=0$, we also obtain the same conclusion.

(iii) Consider the inequality

$$
\begin{aligned}
& \ln (t+1) \int_{0}^{t}\|U(t-s)\|\|f(s+1,0)\| d s \\
& \leqq \int_{0}^{t}\{|\ln (t-s)|+\ln (s+1)+\ln 2\}\|U(t-s)\|\|f(s+1,0)\| d s .
\end{aligned}
$$

Here we have used the inequality 


$$
|\ln (x+y)| \leqq|\ln x|+|\ln y|+\ln 2, \quad x>0, \quad y>0 .
$$

It follows from (3.16) that the right-hand side of $(3.24)$ is bounded on $(0, \infty)$. Therefore the estimate (3.17) follows from the following inequality:

$$
\begin{aligned}
& \ln (t+1)\|x(t+1)\| \\
& \leqq \ln (t+1)\|U(t) x(1)\|+\ln (t+1) \int_{0}^{t}\|U(t-s)\|\|f(s+1,0)\| d s \\
& \quad+\int_{0}^{t}\{|\ln (t-s)|+\ln (s+1)+\ln 2\} K\|U(t-s)\| ! \mid x(s+1) \| d s .
\end{aligned}
$$

\section{Asymptotic behavior II}

Q.E.D.

In this section it is assumed that $f(t, x)$ satisfies (1.7). Furthermore it is assumed that $\lambda>0$ in (1.5) and that for the simplicity $k(c)$ satisfies

$$
k(c) \leqq K c^{a}, \quad c>0,
$$

where $K>0$ and $a>0$ are some constants. Then the following lemma holds:

Lemma 4.1. Suppose that $f(t, x)$ satisfies (1.7) and (4.1) and that the estimate

$$
\begin{aligned}
p=\frac{a}{a+1}\left[(a+1) K M \lambda^{a-1} \Gamma(1-\alpha)\right]^{-1 / a}-M \lambda^{a-1} \Gamma(1-\alpha) \\
\sup _{t \geqq 0}\|f(t, 0)\|>0
\end{aligned}
$$

holds. Then the global solution $x(t)$ of (2.1) uniquely exists for $x_{0} \in D(A)$ satisfying

$$
\left\|A x_{0}\right\| \leqq p\left[\left\|A^{-1}\right\|+M \lambda^{\omega-1} \Gamma(1-\alpha)\right]^{-1},
$$

and satisfies the estimate

$$
\|x(t)\| \leqq\left[(a+1) K M \lambda^{\sigma-1} \Gamma(1-\alpha)\right]^{-1 / a}, \quad t \geqq 0 .
$$

Proof. In the successive approximations (2.2), let

$$
e_{n}=\sup _{t \geqq 0}\left\|x_{n}(t)\right\|
$$

Since $x_{n}(t)$ satisfy the estimates

$$
\left\|x_{n+1}(t)\right\| \leqq\left\|U(t) x_{0}\right\|+\int_{0}^{t}\|U(t-s)\|\left\{k\left(e_{n}\right)\left\|x_{n}(s)\right\|+\|f(s, 0)\|\right\} d s,
$$

it follows that the estimates

$$
e_{n+1} \leqq e_{0}+M \lambda^{\infty-1} \Gamma(1-\alpha) \sup _{t \geqq 0}\|f(t, 0)\|+K M \lambda^{a-1} \Gamma(1-\alpha) e_{n}^{a+1}
$$

hold for $n \geqq 0$. On the other hand, we obtain

$$
\left\|U(t) A^{-1}\right\| \leqq\left\|A^{-1}\right\|+M \lambda^{\omega-1} \Gamma(1-\alpha), \quad t>0
$$


from the equation

$$
U(t) A^{-1}=A^{-1}+\int_{0}^{t} U(s) d s, \quad t>0 .
$$

It follows from (4.2), (4.3), (4.5), and (4.6) that

$$
\begin{gathered}
e_{n+1} \leqq \frac{a}{a+1}\left[(a+1) K M \lambda^{a-1} \Gamma(1-\alpha)\right]^{-1 / a}+K M \lambda^{a-1} \Gamma(1-\alpha) e_{n}{ }^{a+1}, \\
e_{0}<\left[(a+1) K M \lambda^{a-1} \Gamma(1-\alpha)\right]^{-1 / a} .
\end{gathered}
$$

(4.7) implies that

$$
e_{n}<\left[(a+1) K M \lambda^{\alpha-1} \Gamma(1-\alpha)\right]^{-1 / a}, \quad n \geqq 0 .
$$

Therefore $x_{n}(t)$ converge to $x(t)$ uniformly on any finite closed interval in $[0, \infty)$. Clearly $x(t)$ is the unique solution of $(2.1)$.

If the conditions (2.4) to (2.7) are satisfied, it is clear that $x(t)$ is the unique solution of (1.1) under the conditions in Lemma 4.1. Thus we have arrived at the following assertion:

Theorem 4.2. Suppose that the assumptions of Lemma 4.1 are satisfied and that $f(t, x)$ satisfies (2.4) to (2.7). Then there exists the unique solution $x(t)$ of (1.1) for $x_{0}$ satisfying (4.3) and $x(t)$ satisfies the estimate

$$
\varlimsup_{t \rightarrow \infty}\|x(t)\| \leqq \frac{a+1}{a} M \lambda^{\omega-1} \Gamma(1-\alpha) \varlimsup_{t \rightarrow \infty}\|f(t, 0)\| .
$$

Proof. For any $\varepsilon>0$ choose $t_{0}>0$ so large that the estimates

$$
\|x(t)\| \leqq \varlimsup_{t \rightarrow \infty}\|x(t)\|+\varepsilon, \quad\|f(t, 0)\| \leqq \varlimsup_{t \rightarrow \infty}\|f(t, 0)\|+\varepsilon
$$

hold for $t \geqq t_{0}$. Then for $t>0$ we have

$$
\begin{aligned}
\left\|x\left(t+t_{0}\right)\right\| \leqq & \left\|U(t) x\left(t_{0}\right)\right\|+\int_{0}^{t} \frac{M e^{-\lambda(t-s)}}{(t-s)^{\alpha}}\left\{\frac{1}{(a+1) M \lambda^{\omega-1} \Gamma(1-\alpha)}\right. \\
& \left.\times\left\|x\left(s+t_{0}\right)\right\|+\left\|f\left(s+t_{0}, 0\right)\right\|\right\} d s \\
\leqq & \left\|U(t) x\left(t_{0}\right)\right\|+\frac{1}{a+1}\left\{\varlimsup_{t \rightarrow \infty}\|x(t)\|+\varepsilon\right\}+M \lambda^{\omega-1} \Gamma(1-\alpha) \\
& \times\left\{\varlimsup_{t \rightarrow \infty}\|f(t, 0)\|+\varepsilon\right\} .
\end{aligned}
$$

It follows from (4.10) that

$$
\varlimsup_{t \rightarrow \infty}\|x(t)\| \leqq \frac{1}{a+1}\left\{\varlimsup_{t \rightarrow \infty}\|x(t)\|+\varepsilon\right\}+M \lambda^{\omega-1} \Gamma(1-\alpha)\left\{\varlimsup_{t \rightarrow \infty}\|f(t, 0)\|+\varepsilon\right\} .
$$

Since $\varepsilon>0$ is arbitrary, the above inequality implies that (4.9) holds. Q.E.D. 
Corresponding to Theorem 3.3, the following theorem holds. The proof can be carried out in the same way as that of Theorem 3.3 with some modifications.

Theorem 4.3. Suppose that (1.7) and (4.2) are satisfied. Let $x(t)$ be the solution of (2.1) with $x_{0}$ satisfying (4.3).

(i) If there exist $c>0$ and $\delta>0$ such that the estimate (3.12) holds, then $x(t)$ satisfies the estimate

$$
\|x(t)\| \leqq \tilde{c} e^{-\tilde{\delta} t}, \quad t \geqq 0,
$$

where $\tilde{c}>0$ and $\widetilde{\delta}>0$ are some constants.

(ii) If there exist $c>0$, an integer $n \geqq 0$, and $\beta, 0 \leqq \beta<1$, such that the est $i$ mate (3.14) holds when $t \rightarrow \infty$, then $x(t)$ satisfies the estimate (3.15) when $t \rightarrow \infty$.

(iii) If there exists $c>0$ such that the estimate (3.16) holds when $t \rightarrow \infty$, then $x(t)$ satisfies the estimate (3.17) when $t \rightarrow \infty$.

\section{Asymptotic behavior III}

In this section it is assumed that $f(t, x)$ satisfies (2.4) to (2.7) and either (1.6) or (1.7) and that there exists the unique solution of (1.1).

Theorem 5.1. Suppose that the solution $x(t)$ of (1.1) satisfies the estimate

$$
p=M \lambda^{\alpha-1} \Gamma(1-\alpha) \varlimsup_{t \rightarrow \infty}\|D f(t, x(t))+\bar{D} f(t, x(t))\|_{L(E, E)}<1 .
$$

Then we have the estimate

$$
\varlimsup_{t \rightarrow \infty}\left\|\frac{d}{d t} x(t)\right\| \leqq(1-p)^{-1} M \lambda^{\omega-1} \Gamma(1-\alpha) \varlimsup_{t \rightarrow \infty}\left\|f_{t}(t, x(t))\right\| .
$$

Remark 1. Let $A$ (resp. $B$ ) a linear (resp. anti-linear) bounded operator. Then $\|A+B\|_{L(E, E)}$ is understood to be

$$
\sup _{\|x\| \leqq 1}\|A x+B x\| \text {. }
$$

REMARK 2. Suppose that the estimates

$$
\begin{aligned}
& \|D f(t, x)-D f(t, y)\|_{L(E, E)} \leqq k_{1}(c)\|x-y\|, \\
& \|\bar{D} f(t, x)-\bar{D} f(t, y)\|_{L(E, E)} \leqq k_{2}(c)\|x-y\|
\end{aligned}
$$

hold for $t, x$, and $y$ satisfying $t \geqq 0,\|x\| \leqq c,\|y\| \leqq c$, where $k_{1}(c)$ and $k_{2}(c)$ are monotone non-decreasing functions of $c>0$ which are right continuous. Then the condition (5.1) can be written in the more concrete form by combining the above inequalities with (3.5) or (4.9).

Proof. (5.2) is clear in the case where $\varlimsup_{t \rightarrow \infty}\left\|f_{t}(t, x(t))\right\|=+\infty$, Therefore 
we assume that $\varlimsup_{t \rightarrow \infty}\left\|f_{t}(t, x(t))\right\|<+\infty$. Consider the inequality

$$
\begin{aligned}
\left\|\frac{d}{d t} x\left(t+t_{0}\right)\right\| \leqq & \left\|U(t) \frac{d}{d t} x\left(t_{0}\right)\right\|+\int_{0}^{t}\|U(t-s)\|\left\|f_{s}\left(s+t_{0}, x\left(s+t_{0}\right)\right)\right\| d s \\
& +\int_{0}^{t}\|U(t-s)\| \| D f\left(s+t_{0}, x\left(s+t_{0}\right)\right) \\
& +\bar{D} f\left(s+t_{0}, x\left(s+t_{0}\right)\right)\left\|_{L(E, E)}\right\| \frac{d}{d s} x\left(s+t_{0}\right) \| d s
\end{aligned}
$$

where $t_{0}>0$ and $t>0$. Take any $\varepsilon>0$ so that the inequality

$$
p+M \lambda^{\infty-1} \Gamma(1-\alpha) \varepsilon<1
$$

holds. Next take $t_{0}>0$ so large that the following inequalities hold:

$$
\begin{aligned}
& \sup _{s \geqq t_{0}}\left\|f_{s}(s, x(s))\right\|<\varlimsup_{t \rightarrow \infty}\left\|f_{t}(t, x(t))\right\|+\varepsilon, \\
& \sup _{s \geqq t_{0}}\|D f(s, x(s))+\bar{D} f(s, x(s))\|_{L(E, E)} \\
& \leqq \varlimsup_{t \rightarrow \infty}\|D f(t, x(t))+\bar{D} f(t, x(t))\|_{L(E, E)}+\varepsilon .
\end{aligned}
$$

Then it follows from (5.3), (5.5), and (5.6) that

$$
\begin{aligned}
& \left\|\frac{d}{d t} x\left(t+t_{0}\right)\right\| \leqq\left\|U(t) \frac{d}{d t} x\left(t_{0}\right)\right\|+\int_{0}^{t}\|U(t-s)\|\left\{\varlimsup_{t \rightarrow \infty}\left\|f_{t}(t, x(t))\right\|+\varepsilon\right\} d s \\
& +\int_{0}^{t}\|U(t-s)\|\left\{\varlimsup_{t \rightarrow \infty}\|D f(t, x(t))+\bar{D} f(t, x(t))\|_{L(E, E)}+\varepsilon\right\} \\
& \times\left\|\frac{d}{d s} x\left(s+t_{0}\right)\right\| d s, \quad t>0 .
\end{aligned}
$$

In the same way as Theorem 3.2, the inequalities (5.4) and (5.7) imply that $\left\|\frac{d}{d t} x\left(t+t_{0}\right)\right\|$ is bounded on $[0, \infty)$. If necessary, take $t_{0}>0$ so large that the inequality

$$
\sup _{s \geqq t_{0}}\left\|\frac{d}{d s} x(s)\right\| \leqq \varlimsup_{t \rightarrow \infty}\left\|\frac{d}{d t} x(t)\right\|+\varepsilon
$$

holds. It follows from (5.7) and (5.8) that

$$
\begin{aligned}
& \varlimsup_{t \rightarrow \infty}\left\|\frac{d}{d t} x(t)\right\| \leqq M \lambda^{\infty-1} \Gamma(1-\alpha)\left\{\varlimsup_{t \rightarrow \infty}\left\|f_{t}(t, x(t))\right\|+\varepsilon\right\}+M \lambda^{\infty-1} \Gamma(1-\alpha) \\
& \left\{\varlimsup_{t \rightarrow \infty}\|D f(t, x(t))+\bar{D} f(t, x(t))\|_{L(E, E)}+\varepsilon\right\}\left\{\varlimsup_{t \rightarrow \infty}\left\|\frac{d}{d t} x(t)\right\|+\varepsilon\right\}
\end{aligned}
$$

Since $\varepsilon>0$ is arbitrary, (5.9) implies (5.2).

Next suppose that $f(t, x)$ satisfies the following condition: For each $c>0$, 
there exists a bounded measurable function $k(t, c)$ of $t$ which is integrable on $(0, \infty)$ and $k(t, c)$ satisfies

$$
\begin{gathered}
\left\|f_{t}(t, x)\right\| \leqq k(t, c), \quad\|x\| \leqq c, \quad t>0 \\
\lim _{t \rightarrow \infty} k(t, c)=0 .
\end{gathered}
$$

From (5.10) we find that there exists a continuous function $f_{\infty}(x)$ such that $f(t, x)$ converges to $f_{\infty}(x)$ as $t \rightarrow \infty$ uniformly on each bounded set in $E$.

Theorem 5.2. Suppose that $f(t, x)$ satisfies (5.10). If the solution $x(t)$ of (1.1) is bounded on $[0, \infty)$ and satisfies the estimate (5.1), there exists $x(\infty) \in D(A)$ which satisfies the equation

$$
A x(\infty)+f_{\infty}(x(\infty))=0
$$

and $x(t)$ converges to $x(\infty)$ as $t \rightarrow \infty$.

Proof. Let $\|x(t)\| \leqq c, t \geqq 0$. Then it follows from (5.2) and (5.10) that

$$
\lim _{t \rightarrow \infty}\left\|\frac{d}{d t} x(t)\right\|=0
$$

Take $t_{0}>0$ so large that the inequality

$$
M \lambda^{\alpha-1} \Gamma(1-\alpha) \sup _{s \geqq t_{0}}\|D f(s, x(s))+\bar{D} f(s, x(s))\|_{L(E, E)}<p+\varepsilon<1
$$

holds, where $\varepsilon>0$ is arbitrary.

By integrating the both sides of (5.3) with respect to $t$ from 0 to $T$, we obtain

$$
\begin{aligned}
\int_{0}^{T}\left\|\frac{d}{d t} x\left(t+t_{0}\right)\right\| d t \leqq & M \lambda^{\omega-1} \Gamma(1-\alpha)\left[\left\|\frac{d}{d t} x\left(t_{0}\right)\right\|+\int_{0}^{\infty} k(t, c) d t\right] \\
& +(p+\varepsilon) \int_{0}^{T}\left\|\frac{d}{d t} x\left(t+t_{0}\right)\right\| d t
\end{aligned}
$$

where $T>0$ is arbitrary. Therefore (5.14) implies that

$$
\int_{0}^{\infty}\left\|\frac{d}{d t} x(t)\right\| d t<+\infty
$$

Consequently it follows that there exists $x(\infty) \in E$ such that

$$
x(t) \rightarrow x(\infty), \quad f(t, x(t)) \rightarrow f_{\infty}(x(\infty)) \quad(t \rightarrow \infty) .
$$

From (1.1), (5.12), and (5.16), we obtain $x(\infty) \in D(A)$ and (5.11).

Corresponding to the degree of decreasing of $k(t, c)$, the following theorem holds: 
Theorem 5.3. Suppose that the assumptions of Theorem 5.2 are satisfied.

(i) If for each $c>0$ there exist $d>0$ and $\delta>0$ such that the estimate

$$
k(t, c) \leqq d e^{-\delta t}, \quad t>0
$$

holds, then the solution $x(t)$ of (1.1) satisfies the estimate

$$
\|x(t)-x(\infty)\| \leqq \tilde{d} e^{-\tilde{\delta} t}, \quad t>0,
$$

where $\tilde{d}>0$ and $\tilde{\delta}>0$ are some constants.

(ii) If for each $c>0$ there exist $d>0$, an integer $n \geqq 1$, and $\beta, 0<\beta \leqq 1$, such that the estimate

$$
k(t, c) \leqq d t^{-(n+\beta)}
$$

holds when $t \rightarrow \infty$, then $x(t)$ satisfies the estimate

$$
\|x(t)-x(\infty)\| \leqq \tilde{d} t^{-(n+\beta-1)}
$$

when $t \rightarrow \infty$, where $\tilde{d}>0$ is some constant.

Proof. In the same way as Theorem 3.3, (i), it follows that $e^{\nu t}\left\|\frac{d}{d t} x(t)\right\|$ is bounded when $t \rightarrow \infty$, where $\nu>0$ is some constant. Therefore (5.18) follows from the equation

$$
x(\infty)-x(t)=\int_{t}^{\infty} \frac{d}{d \tau} x(\tau) d \tau, \quad t \geqq 0 .
$$

The proof of (ii) is similar to the above arguments. Hence we omit it. Q.E.D.

Corollary 5.4. Suppose that $f(t, x)$ is independent of $t$. If the solution $x(t)$ of (1.1) satisfies the estimate

$$
M \lambda^{\alpha-1} \Gamma(1-\alpha) \varlimsup_{t \rightarrow \infty}|| D f(x(t))+\bar{D} f(x(t)) \|_{L(E, E)}<1,
$$

then there exists $x(\infty) \in D(A)$ which satisfies the equation

$$
A x(\infty)+f(x(\infty))=0
$$

and $x(t)$ converges to $x(\infty)$ exponentially.

Proof. The proof is carried out in the same way as that of Theorem 5.2 and Theorem 5.3. Hence we omit it.

\section{Examples}

In this section we give some examples of linear partial differential equations whose semigroups satisfy the estimate (1.5). 
Example 1. Consider the initial boundary value problem of the heat equation

$$
\begin{aligned}
& \frac{\partial}{\partial t} u(t, x)=\frac{\partial^{2}}{\partial x^{2}} u(t, x), \quad t>0, \quad 0 \leqq x \leqq 1, \\
& u(0, x)=u_{0}(x), \quad 0 \leqq x \leqq 1, \\
& \frac{\partial}{\partial x} u(t, 0)=\frac{\partial}{\partial x} u(t, 1)=0 .
\end{aligned}
$$

Let $E=C^{\infty}([0,1])$, where $0<\alpha<1$. The norm in $E$ is given by

$$
\|u\|_{\infty}=\sup _{0 \leqq x \leqq 1}|u(x)|+\sup _{0 \leqq x, y \leqq 1} \frac{|u(x)-u(y)|}{|x-y|^{\infty}} .
$$

The operator $A$ and the domain $D(A)$ are given by

$$
A u=\frac{d^{2}}{d x^{2}} u, \quad D(A)=\left\{u \in C^{2+\infty} ; \frac{d}{d x} u(0)=\frac{d}{d x} u(1)=0\right\} .
$$

The resolvent $R(\mu)$ of $A$ exists in the complex plane except for the non-positive real semiaxis and is represented as follows:

$$
\begin{aligned}
& u(x)=R(\mu) f=-\frac{\cosh \sqrt{\mu}(x-1)}{\sqrt{\mu} \sinh \sqrt{\mu}} \int_{0}^{x} \cosh \sqrt{\mu} \xi \cdot f(\xi) d \xi \\
& -\frac{\cosh \sqrt{\mu} x}{\sqrt{\mu} \sinh \sqrt{\mu}} \int_{x}^{1} \cosh \sqrt{\mu}(\xi-1) \cdot f(\xi) d \xi \text {. }
\end{aligned}
$$

Let any $b>0$ and any $\varepsilon, 0<\varepsilon<\pi / 2$, be given. In the following we shall estimate $\|R(\mu)\|_{a s}$ on the sector $\sum$;

$$
\Sigma=\left\{\mu=\zeta+b ;-\frac{\pi}{2}-\varepsilon \leqq \arg \zeta \leqq \frac{\pi}{2}+\varepsilon\right\}
$$

Let $\mu=r e^{i \theta}$ and $\beta=\cos \frac{\theta}{2}$. It is easy to see that the estimate

$$
\|u\|_{0} \leqq \frac{1}{r \beta}\|f\|_{0}
$$

holds, where $\|\cdot\|_{0}$ denotes the supremum norm of $C([0,1])$.

Let $0 \leqq y<x \leqq 1$. Then from (6.6) we have

$$
\begin{aligned}
\frac{|u(x)-u(y)|}{|x-y|^{\infty}} \leqq & \frac{1}{|x-y|^{\infty}}\left|\frac{1}{\sqrt{\mu} \sinh \sqrt{\mu}} \int_{y}^{x} g(\xi ; x, y)\{f(\xi)-f(y)\} d \xi\right| \\
& +\frac{1}{|x-y|^{\infty}}\left|\frac{1}{\sqrt{\mu} \sinh \sqrt{\mu}} \int_{y}^{x} g(\xi ; x, y) d \xi \cdot f(y)\right|
\end{aligned}
$$


where

$$
\begin{aligned}
& +\frac{|\cosh \sqrt{\mu}(x-1)-\cosh \sqrt{\mu}(y-1)|}{|x-y|^{\infty}|\sqrt{\mu} \sinh \sqrt{\mu}|}\left|\int_{0}^{y} \cosh \sqrt{\mu} \xi \cdot f(\xi) d \xi\right| \\
& +\frac{|\cosh \sqrt{\mu} x-\cosh \sqrt{\mu} y|}{|x-y|^{\infty}|\sqrt{\mu} \sinh \sqrt{\mu}|}\left|\int_{x}^{1} \cosh \sqrt{\mu}(\xi-1) \cdot f(\xi) d \xi\right|,
\end{aligned}
$$

$$
g(\xi ; x, y)=\cosh \sqrt{\mu}(x-1) \cdot \cosh \sqrt{\mu} \xi-\cosh \sqrt{\mu} y \cdot \cosh \sqrt{\mu}(\xi-1) .
$$

It is easy to see that the estimate

$$
\text { [the first term of }(6.8)] \leqq \frac{2}{r \beta}\left\{\|f\|_{\infty}-\|f\|_{0}\right\}
$$

holds. Since the equation

$$
\begin{aligned}
\int_{y}^{x} g(\xi ; x, y) d \xi= & \frac{1}{4 \sqrt{\mu}}\left\{\left(e^{\sqrt{\mu}(x-1)}-e^{\sqrt{ } \bar{\mu}(y-1)}\right)\left(e^{\sqrt{\mu} x}-e^{\sqrt{\mu} y}\right)\right. \\
& \left.-\left(e^{\sqrt{\mu}(1-x)}-e^{\sqrt{\mu}(1-y)}\right)\left(e^{-\sqrt{\mu} x}-e^{-\sqrt{\mu} y}\right)\right\}
\end{aligned}
$$

holds, we obtain the estimate

[the second term of (6.8)]

$$
\leqq r^{\infty / 2-1} \frac{2\left\{2^{3 / 2-\infty}\left|\sin \frac{\theta}{2}\right|^{\infty}+\left(\cos \frac{\theta}{2}\right){ }^{\infty}\right\} e^{\sqrt{r} \beta}}{e^{\sqrt{\bar{r}} \beta}-e^{-\sqrt{\bar{r}} \beta}}\|f\|_{0} .
$$

For the third term of (6.8), we obtain

$$
\text { [the third term of }(6.8)] \leqq\left\{2^{3 / 2-\infty}\left|\sin \frac{\theta}{2}\right|^{\infty}+\left(\cos \frac{\theta}{2}\right)^{\infty}\right\} \beta^{-1} r^{\infty / 2-1}\|f\|_{0} .
$$

Similarly we obtain

$$
\text { [the fourth term of }(6.8)] \leqq\left\{2^{3 / 2-\infty}\left|\sin \frac{\theta}{2}\right|^{\infty}+\left(\cos \frac{\theta}{2}\right)^{\infty}\right\} \beta^{-1} r^{\infty / 2-1}\|f\|_{0} .
$$

Therefore (6.7) and (6.9) to (6.12) imply that the estimate

$$
\|R(\mu)\|_{\infty} \leqq \frac{M_{\Sigma}}{|\mu|^{1-\infty / 2}}, \quad \mu \in \Sigma
$$

holds, where $M_{\Sigma}>0$ is some constant. It is easily seen that (6.13) implies (1.2).

REMARK. In this example we can shift the path of the integration in (1.4) from $\Gamma_{q}$ to $\partial \sum$. Therefore the semigroup $U(t)$ of (6.1), (6.2), and (6.3) satisfies the estimate

$$
\|U(t)\| \leqq M e^{\gamma t} t^{-\infty / 2}, \quad t>0
$$


where $M>0$ and $\gamma$ are some constants.

In the following Examples 2, 3, and $4, E$ is considered to be $C^{a}([0,1])$ and $A$ is considered to be $d^{2} / d x^{2}$, respectively.

Example 2. Consider (6.1) and (6.2) under the boundary condition

$$
u(t, 0)=\frac{\partial}{\partial x} u(t, 1)=0
$$

The domain $D(A)$ of $A$ is given by

$$
D(A)=\left\{u \in C^{2+\infty} ; u(0)=\frac{d}{d x} u(1)=0\right\} .
$$

The resolvent $R(\mu)$ of $A$ exists in the same region as in Example 1 and is represented as

$$
\begin{aligned}
u(x)=R(\mu) f= & -\frac{\cosh \sqrt{\mu}(x-1)}{\sqrt{\bar{\mu}} \cosh \sqrt{\mu}} \int_{0}^{x} \sinh \sqrt{\mu} \xi \cdot f(\xi) d \xi \\
& -\frac{\sinh \sqrt{\mu} x}{\sqrt{\bar{\mu}} \cosh \sqrt{\mu}} \int_{x}^{1} \cosh \sqrt{\mu}(\xi-1) \cdot f(\xi) d \xi .
\end{aligned}
$$

In the similar way to Example $1, R(\mu)$ satisfies the estimate (6.13) on $\Sigma$.

Example 3. Consider (6.1) and (6.2) under the boundary condition

$$
\frac{\partial}{\partial x} u(t, 0)=u(t, 1)=0
$$

The domain $D(A)$ of $A$ is given by

$$
D(A)=\left\{u \in C^{2+\infty} ; \frac{d}{d x} u(0)=u(1)=0\right\} .
$$

Then the resolvent $R(\mu)$ of $A$ exists in the same region as in Example 1 and is represented as

$$
\begin{aligned}
u(x)=R(\mu) f= & \frac{\sinh \sqrt{\mu}(x-1)}{\sqrt{\bar{\mu}} \cosh \sqrt{\mu}} \int_{0}^{x} \cosh \sqrt{\mu} \xi \cdot f(\xi) d \xi \\
& +\frac{\cosh \sqrt{\mu} x}{\sqrt{\bar{\mu}} \cosh \sqrt{\mu}} \int_{x}^{1} \sinh \sqrt{\mu}(\xi-1) \cdot f(\xi) d \xi .
\end{aligned}
$$

In the similar way to Example $1, R(\mu)$ satisfies the estimate (6.13) on $\Sigma$.

Example 4. Consider (6.1) and (6.2) under the boundary condition

$$
u(t, 0)=u(t, 1)=0 .
$$

The domain $D(A)$ of $A$ is given by 


$$
D(A)=\left\{u \in C^{2+\infty} ; u(0)=u(1)=0\right\} .
$$

Then the resolvent $R(\mu)$ exists in the same region as in Example 1 and is represented as

$$
\begin{aligned}
u(x)=R(\mu) f= & \frac{\sinh \sqrt{\mu}(x-1)}{\sqrt{\bar{\mu}} \sinh \sqrt{\mu}} \int_{0}^{x} \sinh \sqrt{\mu} \xi \cdot f(\xi) d \xi \\
& +\frac{\sinh \sqrt{\mu} x}{\sqrt{\bar{\mu}} \sinh \sqrt{\mu}} \int_{x}^{1} \sinh \sqrt{\mu}(\xi-1) \cdot f(\xi) d \xi .
\end{aligned}
$$

In the similar way to Example 1, R( $\mu$ ) satisfies the estimate (6.13) on $\Sigma$.

REMARK. For Example 4, more general results have been obtained. Consider the parabolic equation

$$
\begin{aligned}
\frac{\partial}{\partial t} u= & \sum_{|\beta| \leq 2 m} a_{\beta}(x) \frac{\partial^{|\beta|}}{\partial^{\beta_{1}} x_{1} \cdots \partial^{\beta_{n}} x_{n}} u, \quad t>0, \quad x \in \bar{\Omega}, \quad u(0, x)=u_{0}(x), \quad x \in \bar{\Omega}, \\
& \left.\frac{\partial^{|\beta|}}{\partial^{\beta_{1} x_{1} \cdots \partial^{\beta_{n}} x_{n}}} u\right|_{\partial \Omega}=0, \quad|\beta| \leqq m-1,
\end{aligned}
$$

where $\Omega$ is a domain of $R^{n}$ with a sufficiently smooth boundary and the coefficients $a_{\beta}(x)$ are smooth. Let $E=C^{\infty}(\bar{\Omega})$. W. von Wahl [9] obtained the estimate (6.13) in the case where $\Omega$ is bounded. In the case where $\Omega$ is unbounded, $H$. Kielhöfer [3] obtained (6.13).

Example 5 ([4], p. 161). Consider the following initial value problem of a system which is parabolic in the sense of Silov:

$$
\begin{array}{ll}
\frac{\partial}{\partial t} v_{1}=\frac{\partial^{2}}{\partial x^{2}} v_{1}, & \frac{\partial}{\partial t} v_{2}=i \frac{\partial^{3}}{\partial x^{3}} v_{1}+\frac{\partial^{2}}{\partial x^{2}} v_{2}, \quad t>0, \quad x \in R^{1}, \\
v_{1}(0, x)=\phi_{1}(x), & v_{2}(0, x)=\phi_{2}(x), \quad x \in R^{1} .
\end{array}
$$

Let $E=L^{2}\left(R^{1}\right)$. Then the semigroup $U(t)$ of (6.24) satisfies (1.5) with $\alpha=1 / 2$.

Example 6. Consider the following initial value problem of a system which is parabolic in the sense of Šlov:

$$
\begin{aligned}
& \frac{\partial}{\partial t} v_{1}=\Delta v_{1}, \quad \frac{\partial}{\partial t} v_{2}=\left(i \frac{\partial^{3}}{\partial x_{1}^{3}}-\frac{\partial^{2}}{\partial x_{2}^{2}}\right) v_{1}+\Delta v_{2}, \quad t>0, \quad x=\left(x_{1}, x_{2}\right) \in R^{2} \\
& v_{1}(0, x)=\phi_{1}(x), \quad v_{2}(0, x)=\phi_{2}(x), \quad x \in R^{2}
\end{aligned}
$$

where $\Delta$ denotes the Laplacian in $R^{2}$. Let $E=L^{2}\left(R^{2}\right)$. By the Plancherel's theorem, (6.25) is equivalent to the following system of ordinary differential equations in $L^{2}\left(R^{2}\right)$ : 


$$
\begin{aligned}
& \frac{d}{d t} \tilde{v}_{1}=-\left(p_{1}^{2}+p_{2}^{2}\right) \tilde{v}_{1}, \quad \frac{d}{d t} \tilde{v}_{2}=\left(p_{1}^{3}+p_{2}^{2}\right) \tilde{v}_{1}-\left(p_{1}^{2}+p_{2}^{2}\right) \tilde{v}_{2}, \quad t>0, \\
& p=\left(p_{1}, p_{2}\right) \in R^{2}, \quad \tilde{v}_{1}(0, p)=\tilde{\phi}_{1}(p), \quad \tilde{v}_{2}(0, p)=\tilde{\phi}_{2}(p), \quad p \in R^{2},(6.26)
\end{aligned}
$$

where $\widetilde{\phi}(p)$ denotes the Fourier transform of $\phi(x) \in L^{2}\left(R^{2}\right)$. The semigroup $U(t)$ of (6.26) is the bounded operator of multiplication by the matrix $U(t ; p)$

$$
U(t ; p)=\left[\begin{array}{cc}
e^{-\left(p_{1}^{2}+p_{2}^{2}\right) t} & 0 \\
t\left(p_{1}^{3}+p_{2}^{2}\right) e^{-\left(p_{1}^{2}+p_{2}^{2}\right) t} & e^{-\left(p_{1}^{2}+p_{2}^{2}\right) t}
\end{array}\right] .
$$

$\|U(t)\|$ is calculated according to the formula [4]

$$
\|U(t)\|=\sup _{p \in R^{2}}\|U(t ; p)\|_{2}, \quad t>0,
$$

where $\|U(t ; p)\|_{2}$ is the norm of the matrix $U(t ; p)$ as an operator in $R^{2}$. As is easily seen, the formula (6.28) implies that $U(t)$ satisfies the estimate (1.5) with $\alpha=1 / 2$.

Acknowledgment. The authors wish to thank Professor H. Tanabe for his many helpful suggestions.

OSAKA UNIVERSITY

\section{References}

[1] E. Hille and R.S. Phillips: Functional analysis and semigroups, American Mathematical Society, Providence, R.I., 1957.

[2] T. Kato: Nonlinear evolution equations in Banach spaces, Proc. Symp. Appl. Math. 17 (1965), 50-67.

[3] H. Kielhöfer: Halbgruppen und semilineare Anfangs-Randwertprobleme, Manuscripta Math. 12 (1974), 121-152.

[4] S.G. Krein: Linear differential equations in Banach space, American Mathematical Society, Providence, R.I., 1971.

[5] A. Pazy: A class of semilinear equations of evolution, Israel J. Math. 20 (1975), 23-36.

[6] I.E. Segal: Non-linear semi-groups, Ann. of Math. (2) 78 (1963), 339-364.

[7] P.E. Sobolevskii and V.A. Pogorelenko: Hyperbolic equations in Hilbert space, Siberian Math. J. 8 (1967), 123-145.

[8] H. Tanabe: Evolution equations, Iwanami, Tokyo, 1975 (in Japanese).

[9] W. von Wahl: Gebrochene Potenzen eines elliptischen Operators und parabolische Differentialgleichungen in Räumen hölderstetiger Funktionen, Nachr. Akad. Wiss. Göttingen, II. Math. Phys. Kl. Jg. 1972, 231-258. 